\section{NAZIONALITÀ COLONIALE, ALTERITÀ INDIGENA. LA \\ RICOSTRUZIONE IDENTITARIA NELLA LETTERATURA AUTOBIOGRAFICA AUSTRALIANA DELLE DONNE}

COLONIAL NATIONALITY, ABORIGINAL ALTERITY. RECONSTRUCTION OF THE IDENTITY IN AUTOBIOGRAPHICAL AUSTRALIAN FEMALE LITERATURE

Francesca Di Blasio Università di Trento

\section{RIASSUNTO:}

ABSTRACT:

Gli scritti autobiografici di Della Walker, Sally Morgan, Nugi Garimara di seguito analizzati contribuiscono a formare e informare il concetto di "dissemi-nazione aborigena" (Bhabha), ossia di una entità politica, sociale (B) e che si narra dai margini in cui duecento anni di dominazione bianca l'hanno relegata. Si tratta di una voce nativa e autoriflessiva che offre uno sguardo inedito sui concetti di "cittadinanza", "nazione", "letteratura nazionale"

\section{Parole chiave:}

Postcolonial, Citizenship, Australia, Autobiography, Women writing
Le origini dell'Australia moderna come colonia dell'impero britannico ufficialmente fondata nel 1788 poggiano su un assunto che simbolicamente, se non legalmente, nega la presenza degli Indigeni sul suolo australiano. La colonizzazione ha inizio infatti sulla base del fatto che il subcontinente australiano è "terra nullius", ${ }^{1}$ locuzione che implica non l'assenza effettiva di popolazione ma di una 'sovranità riconosciuta', vale a dire espressa anch'essa nei modi e nei sensi propri della prospettiva eurocentrica e quindi, essenzialmente, imperialista e coloniale. Questo riconduce al paradigma e al paradosso di una situazione strettamente interrelata con le premesse e gli esiti del colonialismo, che negano la sovranità su un territorio a un popolo che lo ha abitato secondo il proprio sistema di leggi, usi e costumi per quarantamila anni almeno, prima dell'arrivo degli invasori.

In quanto si dirà qui di seguito, il concetto di cittadinanza intersecherà quello $\mathrm{d} i$ nazionalita, inteso come versante culturologico del concetto più propriamente giuridico, nel tracciare una ricostruzione identitaria, complessa, composita e differenziata, della First Nation australiana, occorsa a partire soprattutto dalla seconda metà del secolo scorso anche in virtù della produzione artistica e letteraria indigena, un fenomeno con un ruolo assolutamente dirimente in quella medesima ricostruzione.

Dagli anni Sessanta del Novecento, infatti, gli indigeni australiani partecipano al più vasto movimento di rivendicazione dei diritti delle minoranze (non solo) razzial che attraversa il globo. Le lotte politiche e sociali che conducono al riconoscimento della presenza aborigena sul territorio australiano si legano al lento processo di (ri) affermazione dell'identità etnica e culturale degli aborigeni stessi. Questa si attua anche e soprattutto mediante il ricorso a modalità espressive artistiche e letterarie mimesi e interpretazione de cambiamenti in corso (Di Blasio, 2005).

Nell'ambito delle varie forme di espressività artistica, si assiste all'affermarsi di una vera e propria letteratura aborigena - che pure già da diversi decenni andava formandosi nei presupposti ibridati della modernità. Questa letteratura autoctona australiana usa la lingua del dominatore per offrire uno sguardo altro su un territorio, il proprio, abitato da tempo immemore e si serve dei mezzi della lingua egemone, a volte con acuta consapevolezza del paradosso e della problematicità insiti in questo uso linguistico, allo scopo di poter aprire una finestra sufficientemente ampia su una cultura millenaria sabotata e umiliata dall'invasore bianco, in un tempo forse anche troppo recente rispetto alla durata della propria storia passata.

Una trattazione di questo genere di letteratura sollecita proprio un tentativo di definizione, tutt'altro che facile, di "letteratura nazionale", e, a monte, del concetto

1 Si veda Mabo. The Native Title Revolution,

<http://www.nfsa.gov.au/digitallearning/mabo/tn_01.shtml>. Internet. 5.2.2015 
stesso di "nazione", nella consapevolezza che su ciascuno di questi aspetti esiste un dibattito teorico, etico e politico vasto e di profonda attualità.

In prima istanza, è necessario rilevare che le "literatures in English" non coincidono affatto con l'idea di "letteratura nazionale". Quest'ultima viene anzi, per l'ennesima volta, decostruita e problematizzata, dal momento che l'identità (della matrice) linguistica, che dovrebbe determinare la definizione stessa di "letteratura nazionale", produce discorsi (anche in senso foucaultiano) che sono propri di due culture diversissime, quali appunto quelle degli indigeni e dei colonizzatori.

In secondo luogo, su questi presupposti, appare opportuna una ri-definizione dell'idea di "nazione", idea che, come ricorda Homi Bhabha nel suo famosissimo The Location of Culture (1994), è relativamente recente. Riprendendo il saggio di Ernest Renan del 1882 What Is a Nation?, che pone la nascita dell'idea di stato-nazione come risultato della caduta dei grandi imperi della classicità e del Medioevo, Bhabha sottolinea che la "nazione" non è affatto un'entità 'naturale' e 'atavica', bensì una costruzione storicosociale, che proprio in quanto tale è soggetta a un continuo processo di cambiamenti che possono essere anche radicali. Va inoltre sottolineato il fatto che uno dei limiti dell'idea di nazione è che essa presta il fianco alla strumentalizzazione ideologica dei 'nazionalismi', che tendono a cristallizzare le caratteristiche di omogeneità politica, culturale e linguistica della nazione stessa in una dimensione astorica e inviolabile, ovviamente incondivisibile, e più che mai inadatta ad esprimere lo stato delle cose nella post-modernità. Nel senso di un ripensamento dell'idea 'classica' di nazione, e contro le sue degenerazioni, si pone la proposta avanzata da Bhabha del concetto $\mathrm{d}$ "dissemi-nation", ridefinizione che risulta particolarmente adatta al caso dell' Australia.

Quella che si (ri)afferma in Australia a partire dagli anni Sessanta è infatti identificabile come "dissemi-nazione" aborigena, entità politica, sociale e culturale che narra e che si narra dai margini in cui duecento anni di dominazione bianca $l^{\prime}$ hanno relegata, offrendo così uno sguardo inedito sulla realtà dal punto di vista $\mathrm{d}$ una civiltà estremamente complessa e non omologabile neppure al proprio interno. Il mezzo linguistico usato, che è l'inglese o le sue varietà di Nation Language, secondo la definizione che Brathwaite (1995) dà dell'inglese caraibico, è l'unico aspetto che la produzione letteraria aborigena condivide con la cultura anglosassone, insieme all'utilizzo, in forma ibridata, di certi generi letterari tipici della tradizione occidentale. Per il resto, la letteratura aborigena australiana si distingue come fenomeno esclusivo e particolare che, nelle sue molteplici ibridazioni, dà luogo alla (ri)scrittura di una storia recente e spesso rimossa, rappresentandone percorsi, svolte, nodi problematici e rivendicazioni politiche, sociali e culturali (Di Blasio, 2005; Brewster, 1995). Su tale "dissemi-nazione" e sulla/e sua/e rappresentazione/i letteraria/e si soffermerà lo sguardo documentario e critico nelle pagine che seguono, declinato nella prospettiva assunta da alcune autrici indigene australiane contemporanee. L'effetto di questa prospettiva 'dai margini', infatti, si intensifica quando artefici e protagoniste delle narrazioni aborigene sono le donne che affidano le proprie storie di "native as/and other" (Di Blasio, 2005b) al racconto autobiografico. Assistiamo così ad un fenomeno che, a partire dalla sua ricorsività, si può definire un vero e proprio evento culturale, ossia una messa in scena del 'ritorno di un rimosso antropologico'. Significativi dal punto di vista letterario, oltre che culturale, appaiono in tal senso i casi di Della Walker, Sally Morgan, Alice Nannup, delle quali si intende qui discutere.

Tra le numerose opere della tradizione aborigena scritte in risposta all'esperienza della colonizzazione, e che non esitiamo qui a definire 'coloniali', troviamo Me and You: the Life Story of Della Walker, pubblicato nel 1989. La struttura del testo è costruita lungo due linee narrative principali, che si intersecano tra loro: quella del resoconto da testimone oculare riportato dall'autrice, Della Walker, e registrato da Tina Coutts, e quella di altri testimoni, bianchi e neri, amici o datori di lavoro. Il tono candido e sincero di Walker riesce a creare un doppio ordine di significati per il lettore sensibile e attento, il cui interesse per la letteratura aborigena vada oltre un superficiale e scontato senso di political correctness: da un lato abbiamo l'esplicito invito a forme di cooperazione tra bianchi e Aborigeni; dall'altro, il testo stesso fornisce la prova del fatto che una tale interazione sociale pacifica è fallita, almeno finora, nella storia australiana. La cooperazione, allora, viene sì data come una necessità, ma in nessun modo come un compito facile da assolvere, o come una buona intenzione sufficiente a se stessa.

Particolarmente interessante, in questo testo, l'articolazione del rapporto con il territorio australiano, una delle questioni più dolorose e difficili relativamente all'articolazione del rapporto tra dominatori e dominati, dal momento che la storia coloniale d'Australia coincide con quella dell'esproprio della terra ai suoi abitanti originari. La prima, storica sentenza che riconosce il diritto nativo sul territorio australiano, la Mabo Decision, risale al 1992; in essa la Corte Suprema australiana riconosce il diritto di possesso su determinati territori da parte degli indigeni, nel caso specifico nella persona di Eddie Mabo nel procedimento Mabo vs. Queensland, contraddicendo così finalmente il presupposto coloniale originario dell' Australia come "terra nullius".

Le considerazioni sulla questione della terra hanno negli scritti di Walker un ruolo significativamente accusatorio, e allo stesso tempo liberatorio. Dalla fine degli anni Sessanta, Walker ha partecipato alla lotta politica per i diritti aborigeni sul territorio. Scrive in proposito:

È meraviglioso trovarsi in un gruppo di persone che discutono della terra, la nostra terra. Siamo fortunati se riusciamo ad ottenerne un piccolo pezzo. Non chiediamo di più. In origine era nostra comunque. Tutto questo comprare e vendere, è difficile per 
noi da capire. Nel passato la nostra gente non abusava mai della terra. (Walker,1989:

71. Corsivi aggiunti. Traduzione di chi scrive)

Questa breve citazione contiene diverse implicazioni importanti per la letteratura dell'identità aborigena: innanzitutto, il senso proprio degli aborigeni di appartenenza alla terra versus il concetto bianco di possesso della terra e di sua riduzione a bene immobile e commerciabile. La seconda implicazione riguarda l'esproprio illegittimo della terra da parte degli invasori, e la terza l'origine mitica della cultura aborigena e i conseguenti diritti degli indigeni stessi sulla terra. L'espressione "in the beginning" costituisce infatti un topos religioso che assume inevitabilmente connotazioni mitiche che proclama la sacralità della relazione degli aborigeni con la terra e asserisce, su questa base epistemica, un concetto del tutto particolare e inedito per la prospettiva eurocentrica di 'nazionalità' e 'cittadinanza'.

Passiamo ora a un altro testo, quello forse più famoso tra quelli qui presentati, il romanzo autobiografico My Place (1987) di Sally Morgan. Autrice australiana di origini aborigene, Morgan narra la storia della ricostruzione del proprio passato mulbas, ${ }^{2}$ un passato violentemente negato dalla cultura dei colonizzatori e quindi rimosso nella coscienza delle figure parentali del personaggio principale. Le persone affettivamente più vicine alla protagonista hanno sostituito infatti il comune passato aborigeno con l'(auto)attribuzione di una presunta origine indiana, a sua volta scolorita ormai dall'appartenenza, seppur in una posizione di marginalità, alla società dell'Australia degli anni ‘50-'60. Il testo ricostruisce il passato negato e ne ristabilisce le linee portanti, in un percorso ricco di agnizioni identitarie culturali e 'nazionali' (Di Blasio, 2005).

My Place, pubblicato certo non casualmente nel 1987, cioè un anno prima delle celebrazioni per il bicentenario del dominio coloniale in terra australiana, ha dato visibilità e ascolto ad una voce dai margini, - e da margini radicali, essendo geografici, di razza, di classe, di genere, - una voce che tra l'altro ha costituito un vero e proprio caso letterario. Il romanzo ha infatti raggiunto in modo del tutto inaspettato i vertici delle classifiche di vendita, con livelli di tiratura da vero e proprio best seller.

La storia narrata nel testo si struttura in quattro momenti diversi, che vedono le testimonianze raccolte dall'autrice sulle vicende di Arthur, Gladys e Daisy Corunna affiancarsi al racconto della propria esperienza e dei rapporti tra la scrittrice e le persone che diventano i suoi personaggi. Ciò che appare più rilevante, al di là del mero livello organizzativo del testo, è il suo trasporre in scrittura la vita di tre generazioni di donne, le cui vicende sono segnate da un processo di omologazione

2 Il termine più noto tra quelli con i quali gli aborigeni d'Australia hanno preso a denominare se stessi, rigettando la denominazione loro attribuita dalla cultura dominante degli invasori-colonizzatori, è probabilmente "koori", che ha il significato di "persona", "essere umano" nel dialetto degli aborigen della zona sud-orientale. Il termine "mulbas", che ha il medesimo significato, è invece quello usato dagli aborigeni della zona nord-occidentale, della quale si parla nel romanzo di Morgan. a una cultura estranea e prevaricatrice. Su questa base di partenza, come si è detto, il testo registra al proprio interno molteplici 'agnizioni identitarie', ed è in questa chiave che i parallelismi narrativi più stimolanti possono pienamente realizzarsi a vari livelli del discorso. L'omologazione delle protagoniste si configura cioè come alienazione (e quando la lettrice/il lettore lo capisce si è compiuta un'agnizione fondamentale nella sua coscienza estetica ed etica). Quello che voleva essere un processo di "integrazione" forzata diventa, per chi lo subisce, un percorso verso la perdita di identità, di cultura e di linguaggio (Langton, 1977).

L'autrice scrive da "donna, native [e] 'altra"', "con incertezza e necessità" (Minh-ha, 1989: 8. Traduzione di chi scrive), costruendo delle storie che si intersecano con altre più vaste e all'interno delle quali "il movimento che si crea tra la donna che scrive e quella descritta è infinito" (Minh-ha, 1989: 30. Traduzione di chi scrive)

L'agnizione centrale all'interno del testo è indubbiamente quella in base al quale la 'Nazione Australia' si trasforma nella - per tornare all'interessante terminologia d Homi K. Bhabha - (dissemi)nazione aborigena. È questo il fulcro intorno al quale l'essere “donna, nativa e 'altra'” della voce narrante si interseca, in un quadro più ampio, con la storia della gente del vastissimo deserto australiano. La voce narrante nomina la cultura e il passato degli aborigeni, ne diventa memoria e ne è a sua volta chiaramente modificata. Da questo passaggio, che è un autentico scambio culturale ed esistenziale, derivano la costruzione e l'acquisizione, dolorose e allo stesso tempo felici, di una nuova identità (non solo) etnica e nazionale, che si sostanzia proprio a partire dalla scoperta delle proprie autentiche radici negate. Il recupero di quanto la cultura egemone ha voluto cancellare diventa allora il senso più profondo della scrittura, poiché la storia narrata non cessa mai di rimandare, tramite la testimonianza, alla storia vissuta.

Chiude la nostra carrellata Under the Wintamarra Tree, romanzo del 2002 di Nugi Garimara, alias Doris Pilkington, che è il racconto di un ritorno alle origini, dopo l'esperienza della deportazione e dell'alienazione cui i nativi sono stati condannati dal regime coloniale nella loro stessa terra.

Gli antenati dell'autrice, i Mardu della Western Australia, erano stati forzatamente allontananti dalle loro zone d'origine, nel deserto occidentale, e costretti nelle riserve 'bianche' ai suoi margini. La madre di Garimara, Molly, riuscì nell'impresa epica di una incredibile contro-migrazione, che, dopo la fuga dal Moore River Native Settlement, dove era stata internata in seguito ad un vero e proprio rapimento dalla famiglia, ed un lungo e difficile viaggio attraverso il deserto, la riportò, insieme alla sorella minore, nella terra d'origine. Questa vicenda è vividamente narrata in un precedente romanzo di Garimara, Follow the Rabbit-Proof Fence (Garimara, 1996), uno tra i testi più noti della letteratura aborigena, da cui è stato tratto un film, Rabbit-Proof Fence, diretto da Phillip Noyce, di grande risonanza internazionale. In Under the Wintamarra Tree Garimara 
racconta in certo senso il seguito della storia della madre, ricostruendo poi il percorso della nascita della consapevolezza delle proprie radici aborigene.

L'esperienza identitaria aborigena è straordinariamente ben rappresentata, nella sua intrinseca complessità, nel romanzo, che si apre con il racconto di una forzata migrazione, di sradicamento dal proprio territorio. Il titolo del primo capitolo è infatti "Leaving the Desert" e le sue pagine inaugurano il racconto dell'esperienza dolorosa dell'esodo aborigeno, un evento che, diversamente da quello biblico, si traduce presto in schiavitù, anziché in liberazione, per migliaia di indigeni, nella cancellazione di un popolo, anziché nell'affermazione della sua esistenza. La perdita del territorio 'nazionale' coincide, nella sensibilità aborigena, con la perdita dell'identità originaria. Da qui la necessità della protagonista di questo Bildungsroman aborigeno di ritrovare, molti anni dopo, e dopo innumerevoli prove e sofferenze, l'albero di wintamarra sotto il quale era stata partorita. L'albero ha connotazioni sacrali nella cultura della comunità ed è letteralmente l'albero della vita (Di Blasio, 2005).

Ci sono altri aspetti rilevanti rispetto alla definizione della dissemi-nazione aborigena che il romanzo evidenzia fin dall'inizio, in particolare la dimensione nomadica e conservazionista della tradizione locale, nonché l'importanza del ruolo matriarcale nel farsene interprete e nel trasmettere le conoscenze di tempi e spazi alle nuove generazioni. Significativo in tal senso è il brano che segue:

Bambaru era la prima moglie di Tjirama, e la madre delle sue figlie Bami Burungu e Togoda Burungu e dei suoi figli Balga Burungu, Toby Burungu e Ngidi (l'ultimo). .... Bambaru era cieca- aveva persola vista, come molti abitatori del deserto, a causa del tracoma o 'cecita della sabbia' - ma la sua memoria e la sua conoscenza della raccolta e della preparazione del cibo la rendevano di insostituibile importanza. Da bambina aveva vissuto a Buungul, dalle parti del fiume Rudall, con la sua famiglia, che si muoveva in continuazione nel deserto in cerca di cibo, e così aveva imparato molto della terra dei Gududjara. Comprendeva l'importanza di una profonda conoscenza della terra e comunicava molto del suo sapere alle donne più giovani, perché sapeva che sarebbe stato d'aiuto nell'assicurare la sopravvivenza dei suoi figli e dei suoi nipoti. Essi sarebbero stati coloro che avrebbero praticato e preservato i costumi dei Gududjara.

I Gududjara ei Mandildjara avevano vissuto in armonia con la loro terra per migliaia di anni, e, come altri indigeni, erano conservazionisti, prendevano solo quello di cui avevano effettivamente bisogno. Avevano imparato dagli Spiriti ancestrali che se si prendevano cura della terra, la terra avrebbe ricambiato, fornendo loro piante e selvaggina in abbondanza.

Uuesta reciprocità e i suoi obblighi venivano attentamente rispettati non solo dai Gududjara e dai Mandildjara, ma anche dai Mardudjara di Jigalong e oltre, [dai

\footnotetext{
3 Per una esauriente trattazione antropologica di questo aspetto si veda Tamisari 1999.
}

popoli che vivevano] attraverso tutta la vastità e l'estensione della regione del Deserto Occidentale. (Garimara, 2002: 2-3. Traduzione di chi scrive)

Il ritorno alle origini consiste in effetti nel sapersi riappropriare della propria tradizione, recuperando il senso dei ritmi stagionali e dei vasti percorsi spaziali da essi scanditi. Le pratiche stanziali e 'nazionali' dei colonizzatori, che si fissano sul territorio, spesso senza alcun interesse ad esplorarlo, ma recintandolo, delimitandolo per difendere la proprietà dai conigli che, come i nativi, non conoscono limiti nei propri percorsi, si contrappongono a quelle nomadiche e 'dissemi-nazionali' degli indigeni. Come in Follow the Rabbit Proof Fence, il loro senso di "casa" è legato a un territorio sconfinato.

\section{Rifermienti bibliografici}

Bhabha, H., The Location of Culture, London \& New York, Routledge, 1994.

Brathwaite, E.K., Nation Language, in The Postcolonial Studies Reader, Bill Ashcroft, Gareth Griffiths and Helen Tiffin (eds.), London, Routledge, 1995.

----, Rights of Passage, London \& New York, Oxford University Press, 1967.

Brewster, A., Literary Formations: Post-Colonialism, Nationalism, Globalism, Carlton South, Vic., Melbourne University Press, 1995.

Di Blasio, F., The Pelican and the Wintamarra Tree: voci della letteratura aborigena australiana Trento, Università degli Studi di Trento, Collana Labirinti, 2005.

----, “Nativeness and/as Otherness: The Female Gaze in Autobiographical Aboriginal Writings", in G. Buonanno, M. Silver (eds.), Cross-Cultural Encounters: Identity, Gender, Representation, Roma, Officina, 2005b, p. 97-108.

Garimara, N./Doris Pilkington, Under the Wintamarra Tree, St. Lucia, Qld., University of Queensland Press, 2002.

----, Follow the Rabbit Proof Fence, St. Lucia, Qld., University of Queensland Press, 1996

Langton, M., "Self-determination as Oppression", in Australia's Policy Towards Aborigines 1967-1977, Minority Rights Group, Report 351977.

Minh-ha, Trinh T., Woman, Native, Other. Writing Postcoloniality and Feminism, Bloomington, Indiana University Press, 1989.

Morgan, S., My Place, Melbourne, Fremantle Arts Centre Press, 1987.

Tamisari, F., “L'immagine dell'orma. Della cosmogonia indigena”, Quaderni di semantica, XX, 2 (1999), pp. 281-310.

Walker, D., Me and You: the Life Story of Della Walker / as Told to Tina Coutts, Canberra, Aboriginal Studies Press, 1989. 